Epidemiology and Psychiatric Sciences

\section{cambridge.org/eps}

\section{Original Article}

Cite this article: Narzisi A et al (2020). Prevalence of Autism Spectrum Disorder in a large Italian catchment area: a school-based population study within the ASDEU project. Epidemiology and Psychiatric Sciences 29, e5, 1-10. https://doi.org/10.1017/

S2045796018000483

Received: 10 May 2018

Revised: 27 July 2018

Accepted: 1 August 2018

\section{Key words:}

Autism; child psychiatry; diagnosis;

epidemiology; population survey

Author for correspondence:

Sara Calderoni, E-mail:

sara.calderoni@fsm.unipi.it (c) The Author(s) 2018. This is an Open Access article, distributed under the terms of the Creative Commons Attribution licence (http:// creativecommons.org/licenses/by/4.0/), which permits unrestricted re-use, distribution, and reproduction in any medium, provided the original work is properly cited.

\section{CAMBRIDGE UNIVERSITY PRESS}

\title{
Prevalence of Autism Spectrum Disorder in a large Italian catchment area: a school-based population study within the ASDEU project
}

A. Narzisi $^{1}$, M. Posada
, F. Barbieri
, N. N. Chericoni ${ }^{1}$, D. Ciuffolini
Romano

${ }^{1}$ IRCCS Fondazione Stella Maris, Pisa, Italy; ${ }^{2}$ IIER \& CIBERER Instituto de Salud Carlos III, Madrid, Spain; ${ }^{3}$ Child Neuropsychiatry Unit, ASL 5, Pisa, Italy; ${ }^{4}$ Local Education Authority, Firenze, Italy; ${ }^{5}$ Research Coordination and Support Service, Istituto Superiore di Sanità, Rome, Italy and ${ }^{6}$ University of Pisa, Pisa, Italy

\section{Abstract}

Aims. This study aims to estimate Autism Spectrum Disorders (ASD) prevalence in schoolaged children in the province of Pisa (Italy) using the strategy of the ASD in the European Union (ASDEU) project.

Methods. A multistage approach was used to identify cases in a community sample $(N=$ 10 138) of 7-9-year-old children attending elementary schools in Pisa - Italy. First, the number of children with a disability certificate was collected from the Local Health Authority and an ASD diagnosis was verified by the ASDEU team. Second, a Teacher Nomination form (TN) to identify children at risk for ASD was filled in by teachers who joined the study and the Social Communication Questionnaire (SCQ) was filled in by the parents of children identified as positive by the TN; a comprehensive assessment, which included the Autism Diagnostic Observation Schedule-Second Edition, was performed for children with positive TN and $\mathrm{SCQ} \geqslant 9$.

Results. A total of 81 children who had a disability certificate also had ASD (prevalence: $0.79 \%$, i.e. $1 / 126)$. Specifically, 66 children ( 57 males and nine females; $62 \%$ with intellectual disability -ID-) were certified with ASD, whereas another 15 (11 males and four females; $80 \%$ with ID) were recognised as having ASD among those certified with another neurodevelopmental disorder. Considering the population of 4417 (children belonging to schools which agreed to participate in the TN/SCQ procedure) and using only the number of children certified with ASD, the prevalence (38 in 4417 ) was $0.86 \%$, i.e. one in 116 . As far as this population is concerned, the prevalence rises to $1 \%$ if we consider the eight new cases (six males and two females; no subject had ID) identified among children with no pre-existing diagnoses and to $1.15 \%$, i.e., one in 87 , if probabilistic estimation is used.

Conclusions. This is the first population-based ASD prevalence study conducted in Italy so far and its results indicate a prevalence of ASD in children aged 7-9 years of about one in 87. This finding may help regional, national and international health planners to improve ASD policies for ASD children and their families in the public healthcare system.

\section{Introduction}

According to the Diagnostic and Statistical Manual of Mental Disorders, fifth edition (DSM-5) (American Psychiatric Association, 2013), Autism Spectrum Disorders (ASD) are a heterogeneous set of neurodevelopmental disorders characterised by deficits in social communication and social interaction plus the presence of restricted interests and repetitive behaviours.

Since the earliest epidemiological investigations in the 1960s, several independent studies have been conducted to determine the prevalence rates of ASD in various geographic regions, with different methodologies that have generated different results (Hill et al., 2015). A recent systematic review of worldwide studies found a median prevalence of $0.6 \%$ (Elsabbagh et al., 2012). In the USA, the Autism and Developmental Disabilities Monitoring Network (ADDM) has performed multiple studies of ASD prevalence rates starting in 2000 (Centers for Disease Control and Prevention, 2007a, 2007b, 2009, 2012, 2014; Christensen et al., 2016; Baio et al., 2018): the latest estimate of ASD prevalence - one in 59, i.e. $1.7 \%$ - represents an increase of $30 \%$ compared with the one in 88 rate reported in 2008 and an increase of $50 \%$ compared with the one in 150 rate in 2000. The ADDM was conducted in selected US sites and was based on a review of education or healthcare evaluations by trained clinicians. Interestingly, an updated estimate puts ASD prevalence in US children and adolescents at 2.5\% in 2014-2016 (Xu et al., 2018). This estimate derives from the National Health Interview Survey (NHIS) (Parsons et al., 2014), an annual health survey in the USA based on parent report of a physician's diagnosis and conducted on a nationally representative population. However, some 
substantial differences exist between the ADDM and NHIS investigations -including study design, participant characteristics and methods of data gathering - that make it difficult to compare the two reported prevalence rates directly.

More broadly, the heterogeneity in the reported ASD prevalence may be due to several factors: different methodological approaches (e.g. data source), different measures for assessing ASD symptoms (parent and/or teacher questionnaire alone $v$. direct diagnostic assessment) or different criteria to define an ASD, different study designs for case identification (screening of selected population or retrospective chart review) and different characteristics of the populations studied (e.g., age, ethnicity, availability of services, socioeconomic status). Of note, studies involving more strictly defined autistic symptoms, and/or medical records as the data source and/or a wide age-range found low overall prevalence rates of ASD (Davidovitch et al., 2013; Arvidsson et al., 2018; Bachmann et al., 2018). Conversely, the prevalence of ASD resulted higher in studies including less severe forms of ASD, systematic screening of the general school population and/or prevalence restricted to the child population (BaronCohen et al., 2009; Kim et al., 2011; Romhus et al., 2017; Bachmann et al., 2018).

Nevertheless, the increase in ASD prevalence over the years is an established and replicated finding (Rice et al., 2013). The mechanism underlying the rise of ASD prevalence remains poorly understood and certainly involves multiple factors that can be dichotomised in non-aetiologic (i.e. changes in diagnostic and classification criteria, early diagnosis, service availability, inclusion of milder cases, increased public and scientific awareness and identification) and aetiologic (epigenetic, and environmental risk factors).

For example, as far as non-aetiologic factors are concerned, previous investigations found that changes in diagnostic criteria and inclusion of outpatient data played a relevant role in increased ASD prevalence (Hansen et al., 2015; Ramsey et al., 2016). Conversely, the contribution of diagnostic substitution of intellectual disability with ASD first proposed by Croen et al. (2002) to explain the rise in ASD over recent decades has not been confirmed as a relevant factor in a more recent study (Nevison and Blaxill, 2017).

As regards environmental risk factors, a review of systematic reviews and meta-analyses has been conducted recently (Modabbernia et al., 2017). In particular, a positive association between advanced parental age at conception and risk of ASD in offspring was repeatedly found in epidemiological studies (see $\mathrm{Wu}$ et al., 2017, for a systematic review and meta-analysis on this topic). However, it was estimated that approximately only $5 \%$ of the increased prevalence of ASD in a California service system was attributable to older parental age (Shelton et al., 2010). In addition, maternal immune activation (MIA) during pregnancy has been detected as a factor that might increase the risk of ASD (Parker-Athill and Tan, 2010), and in particular, MIA caused by asthma and allergies was associated with an increased severity of social symptoms in the offspring (Patel et al., 2017). The role of other environmental factors in the increased prevalence of ASD remains more controversial: for example, the majority of epidemiological studies on prenatal exposure to air pollutants, pesticides and phthalates indicated an increased risk for ASD (Hertz-Picciotto et al., 2018). However, the different chemical compounds analysed as well as the different exposure metrics used may prevent a direct comparison between studies.
Importantly, further research efforts are needed to better understand and quantify the role played by every single factor in the rise of ASD prevalence as well as their reciprocal influences.

In Europe, the recently reported prevalence of ASD varies considerably between regions and populations. Comparable prevalence rates of ASD were found in Germany (0.4\%; Bachmann et al., 2018), Poland $(0.3 \%$ in children aged 0-16 years; Skonieczna-Żydecka et al., 2016) and France (0.4\% among children aged 7-years old; Van Bakel et al., 2015). In these studies, a possible source of ASD prevalence underestimation is that data were derived from registers (Van Bakel et al., 2015), from government agencies (Skonieczna-Żydecka et al., 2016), or from a German health insurance company (Bachmann et al., 2018), without a systematic screening of new cases. As a consequence, individuals with milder autistic symptoms and higher IQ may not have been included. Conversely, higher prevalence rates were detected in other countries, and specifically: $0.6 \%$ in Belgian children aged 3-39 months (Dereu et al., 2010), 0.7\% in Danish children aged 5-10 years (Parner et al., 2011), 0.9\% in 7-16-year-old children in the Faroe Island (Kočovská et al., 2012); 1.1\% in 6-11-year-old Irish children (Boilson et al., 2016), $1.2 \%$ in Icelandic children aged 11-15 years (Saemundsen et al., 2013); 1.4\% in 0-17-year-old Swedish children (Idring et al., 2015).

Crucially, investigations using the same methodology and conducted in different geographical areas are necessary to make a reliable comparison of ASD prevalence between countries. Moreover, repeated investigations that use the same methodology, but are conducted in the same geographical area at different time points are important to produce more precise information about time trends in the prevalence of ASD (Fombonne, 2009).

As far as Italy is concerned, the limited information available on the prevalence rates of ASD is based on registers of previously ASD-diagnosed subjects aged $0-17$ years. The percentage of ASD cases ranges from values of $0.05 \%$ in the city of Catania Sicily - (Ferrante et al., 2015) to $0.38 \%$ in Piedmont (data obtained from the information system NPI.net, 2016) and $0.39 \%$ in Emilia-Romagna (data from the information system ELEA, 2016).

The aim of the current study was to estimate the prevalence of ASD in a large representative community sample of the child population in Pisa (Italy). The study protocol we adopted is part of a wider project called ASDEU (Autism Spectrum Disorders in European Union). ASDEU was a 3-year program run by a consortium of 20 centres from 14 countries. ASDEU (http://www.asdeu.eu) has received funds from the Directorate-General of Health and Consumers of the European Commission (DG-SANCO) to increase understanding of and improve responses to autism. One of the main aims of ASDEU was the estimation of autism prevalence in 12 countries in the European Union. In this paper, the Italian prevalence study is reported.

\section{Method}

\section{Population}

The target population was composed of 10138 children $(51.6 \%$ males and $48.4 \%$ females), living in the metropolitan area of Pisa. Pisa is located in Tuscany (Central Italy) and the metropolitan area has 182000 inhabitants distributed in the three districts of Pisa, Pontedera and Volterra. The metropolitan area of Pisa has a mean annual income per inhabitant slightly higher than the 
Italian average (D'onofrio and Murro, 2013). The three geographical areas cover a broad cross-section including urban and rural areas. Each of these districts has a Child Neuropsychiatry Service (CNS); moreover, a national reference center for neurodevelopmental disorders (IRCCS Stella Maris Foundation) is located in the Province of Pisa. The IRCCS Stella Maris Foundation is a tertiary care university hospital specialised in the diagnosis of ASD in early childhood: therefore, we could hypothesise that, for children with suspected/confirmed ASD, the likelihood that parents would look for evaluation or treatment outside the Province of Pisa is minimal.

The population was defined as all children living within the metropolitan area of Pisa whose age, during the period of the study, was between 7 and 9 years (selected by birth year: children born between January 1st 2007 and December 31st 2009), irrespective of gender and ethnicity.

According to Italian law (Law 104/1992), children with any type of disability or emotional and behavioural difficulties generally attend mainstream schools, in ordinary classes at all educational levels, from preschool years until the age of 18. In order to receive a support teacher at the school, parents of children with special educational needs must obtain specific certification, issued by the relevant office, after an extensive assessment has been performed by the local CNS to establish the type of diagnosis and level of disability. Curricular teachers are officially informed about the diagnosis of each child. Certification gives access to (a) a functional diagnosis and clinical profile with an analytical description of the pupil's strengths and weaknesses, and developmental possibilities in the short and medium term; (b) an individualised education plan that includes a description of interventions and activities planned for the pupil in a given period. Classes with one or two children with disabilities contain a maximum of 20 pupils. The inclusion process is supported and periodically verified by a project that defines the objectives and strategies adopted jointly by curricular and support teachers. Support teachers are part of the team of regular class teachers and participate in all regular activities; they are also facilitators of all inclusion processes.

According to the ASDEU protocol and considering the inclusion of all children with disabilities in regular Italian schools, two phases of the prevalence study were performed: (1) detection of the number of certified children with a diagnosis of ASD; (2) identification of new cases.

\section{Phases of the field study}

Phase 1. Number of certified children with a diagnosis of ASD The first step of the study was to obtain information from the Local Unit of the Ministry of Education about the number of 7-9-year-old children with a disability who also had a support teacher according to the Italian Law 104/92. Among these, we identified children with a primary diagnosis of ASD already performed by the local CNS and children with a diagnosis of ASD among those who were certified with other disabilities. This first phase was accomplished by means of a clinical consensus between the ASDEU team and the CNS team; the consensus was reached after verifying the reported diagnosis in clinical records from the CNS. The clinical records included measures of Intelligence Quotient (IQ), language and social skills obtained through the administration of the appropriate module of the Autism Diagnostic Observation Schedule-Second Edition (ADOS-2; Lord et al., 2012).
Phase 2. Identification of new cases of ASD by means of a direct school population screening

This second phase was composed of two steps: (a) identification of children at risk for an ASD; (b) clinical assessment of screenpositive children.

\section{All schools within the metropolitan area of Pisa were invited to participate in the study}

In order to increase participation, together with the teacher and parent invitations we included an official letter expressing strong support for the project from the superintendent of schools. Two investigators (A.N. and S.C.) met with teachers and principals to explain the purpose and the importance of the study and encourage their participation.

Two meetings for each district in the metropolitan area of Pisa were organised to describe the study and the instruments. In particular, it was specified that the research team was interested in identifying children with no previous ASD diagnosis. A two-page document describing the study was delivered. The schools that agreed to take part in the study were asked to carry out two tasks: (1) to have teachers fill in the Teacher Nomination form (TN) considering all the 7-9-year-old children in their classes, with the exclusion of certified children; the TN was used as a screening instrument to identify children with no pre-existing ASD diagnosis; (2) to distribute the Social Communication Questionnaire (SCQ) to the parents of children identified by the TN.

\section{TN Form}

The Teacher Nomination Form (Hepburn et al., 2008) contains a list of child characteristics associated with ASD, which were culled from a larger list obtained by brainstorming with clinicians and educators who had extensive experience working with children with autism or with Asperger syndrome. The TN was delivered to teachers who were asked to complete it with regard to the children in their current classes. The TN was estimated to require 2-10 min per class. Participating teachers were provided with the one-page 'Teacher Nomination Form' and asked if any children in their class fit the description, and if so, how many children. Descriptors included the following six items: 'Socially awkward'; 'Doesn't seem to understand the feelings of others'; 'Talks a lot about his/her own interests, but is not very good at conversation'; 'Doesn't really chat to be friendly'; 'Not very flexible-Tends to insist on certain rules and routines'; and 'Is intensely interested in just a few topics or activities'. Then, they were asked to nominate a minimum of two and a maximum of four children in their class who best fit the descriptors on the Nomination Form ranked in order of who fit the description best. The form requested that a minimum of two and a maximum of four children be nominated, regardless of how many children in the class fit the description and even if teachers felt that none of the children really fit the description. The TN strategy was compared with the ASSQ (Autism Spectrum Screening Questionnaire; Ehlers et al., 1999) strategy (which takes about $2 \mathrm{~h}$ per class) by Hepburn et al. (2008), who showed that the proportion of overall agreement between the two measures ranged from 93 to $95 \%$ and that the nomination strategy was more sensitive when teachers were asked to nominate at least two children.

\section{Social Communication Questionnaire}

The SCQ (Rutter et al., 2003a) is a 40-item, parent-report screening measure that taps the symptomatology associated with ASD. 
The items are administered in a yes/no response format and can generally be completed by the parent (or other primary caregiver) in less than $10 \mathrm{~min}$ and scored by the administrator in under 5 min. The SCQ Lifetime (used in this study) is completed with reference to the individual's entire developmental history and produces results that are pertinent to referral for a more complete diagnostic assessment. The authors recommend a comprehensive ASD assessment for all subjects who meet or exceed the cutoff score of 15 . However, there is no consensus regarding the most effective SCQ score, as it varies according to the characteristics of the study (e.g., age, SCQ version, sample features) (Chesnut et al., 2017). Previous studies suggested lowering the SCQ cutoff to 13 (Snow and Lecavalier, 2008), 12 (Corsello et al., 2007), 11 (Allen et al., 2007), 8 or 7 (Schanding et al., 2012), or 7 (Barnard-Brak et al., 2016) in order to enhance the accuracy of this instrument. Thus, to reduce the chance of false negatives, for the purposes of this study, we considered children with a total score of 9 or greater as at risk for ASD, in accordance with the ASDEU protocol.

\section{Clinical assessment of new cases}

Step 2.1 consisted in assessing positive cases (TN positive plus $\mathrm{SCQ} \geqslant 9$ ) in order to confirm or exclude the risk of ASD. The Autism Diagnostic Interview-Revised (ADI-R; Rutter et al., 2003b) was used in combination with the ADOS-2. Both instruments were administered by a member of the research team (A.N.), unaware of the exact SCQ score and trained in the use of the ADI-R and ADOS with a high-reliability level. The ADI-R is a semi-structured interview conducted with a primary caregiver of the individual referred for the evaluation of possible ASD. The interview measures behaviours in three areas: social reciprocity, communication and stereotyped behaviours, as well as onset, based on history for children over age 5. The ADI-R includes a scoring algorithm based on the DSM-IV/ICD-10 criteria for autism that yields a classification of autism or nonautism. In order to achieve an ADI-R classification of autism, an individual must meet cut-offs in each area as well as onset criteria.

The ADOS-2 is a semi-structured, standardised assessment of communication, social interaction, play and restricted and repetitive behaviours. It provides a highly accurate picture of current symptoms, unaffected by language. It can be used to evaluate almost anyone suspected of having ASD from 1-year-olds with no speech, to adults who are verbally fluent. For this study, due to the age and IQ level of subjects, we always used Module 3. This Module is intended for subjects with a spontaneous complex speech which is defined as producing a range of sentence types and grammatical forms, using language to provide information about events out of the context of the ADOS and producing some logical connections within sentences. The revised algorithm was used, which consists of two domains, Social Affect and Restricted, Repetitive Behaviours, combined to one score to which thresholds are applied, i.e., $>7$ for ASD and $>9$ for autism (Gotham et al., 2007). The administration of ADI-R and ADOS-2 was followed by a consensus diagnosis based on information obtained through the ADOS-2 in conjunction with a clinical judgement, according to DSM-5 criteria. Finally, IQ data, measured with the Wechsler Intelligence Scale for Children-Fourth Edition (WISC-IV; Wechsler, 2003) was also collected. The WISC-IV is a scale for assessing cognitive ability, which measures verbal comprehension, perceptual reasoning, working memory and processing speed. After the clinical assessment, only children with a consensus ASD and a total ADOS-2 (Module 3) score $\geqslant 7$ were defined as having ASD.

\section{Ethical permission}

The study protocol (version 1-22-11-16) was approved by the Pediatric Ethics Committee of the Tuscany Region in November 2016 (Approval Number: 178/2016).

\section{Results}

\section{Phase 1. Number of certified children with a diagnosis of ASD}

The data provided by the Local Unit of the Ministry of Education informed us that out of 10138 children (aged 7-9 years) attending schools in Pisa, 239 had a disability certificate. Of these certified children, 66 already had an ASD diagnosis made by the local CNS. Moreover, clinical consensus -based on the analysis of the previous clinical reports and defined as agreement among three clinicians (A.N., F.M., F.B.) - enabled us to identify 15 other children with an ASD diagnosis among those who had a certified diagnosis of other neurodevelopmental disorders (Attention Deficit Hyperactivity Disorder, Intellectual Disability - ID -, Language Disorder, Learning Disorder). This population of 81 (66 plus 15) children of 7-9 years was composed of $68(84 \%)$ males and 13 (16\%) females (male-to-female ratio: 5.2:1). Autism severity (evaluated through the calibrated severity scores CSS -; Gotham et al., 2009) was high for $63 \%$, moderate for $16 \%$, and low for $21 \%$. Performance IQ level was $>75$ for $34 \% ; 41 \%$ had a mild ID (>50 IQ <75) and $25 \%$ had a severe ID (IQ < 50). As far as expressive language is concerned, $52 \%$ of subjects were non-verbal.

Considering that there were 81 children with a confirmed diagnosis of ASD, the ASD prevalence is of $0.8 \%$ (95\% CI $0.62-0.97$ ) that is $1 / 126$.

\section{Phase 2. Identification of new cases at risk of ASD by means of} direct school population screening

In all, 72 out of 160 (45\%) schools joined Phase 2 of the study. The total number of children who took part in Phase 2 was 4417 (43.5\% of the 10138 total number of 7-9-year-old children in the area); of these 4417 children, 111 were certified with disability (Table 1). Thus, in Phase 2, 115 teachers filled the TN Form for 4306 children. Out of these 4306 children, 342 (8\%) were nominated through the TN. This result is in line with data from the original investigation of Hepburn et al. (2008), in which teachers nominated $8.6 \%$ of included children. Parents of these 342 children filled in the SCQ and 49 children (14\%; M: 39, F: 10) out of the 342 nominated by teachers obtained a score $\geqslant 9$. Children scoring $<9$ were not sampled for assessment, in accordance with the ASDEU protocol, shared by 12 countries in the European Union.

The 49 children at risk (TN positive plus SCQ $\geqslant 9$ ) then underwent the clinical assessment procedure. Of these 49 children, ten (20\%; M: 8, F: 2) children obtained an SCQ score $\geqslant 15$ and six of them agreed to be evaluated: all of these children received a diagnosis of ASD. Seventeen children (35\%; M: 12, F: 5) obtained an SCQ score between 11 and 14 , and ten of them accepted the evaluation phase, which resulted in a diagnosis of ASD for one child; 22 children (45\%; M: 19, F: 3) obtained an SCQ score of 9 or 10, and 14 of 
Table 1. Demographic and clinical characteristics of children $(n=81)$ certified by the local health authority

\begin{tabular}{|c|c|}
\hline Gender & $\%(n)$ \\
\hline Males & $84(68)$ \\
\hline Females & 16 (13) \\
\hline \multicolumn{2}{|c|}{ Autism severity ${ }^{a}$} \\
\hline High & $63(51)$ \\
\hline Moderate & 16 (13) \\
\hline Low & 21 (17) \\
\hline \multicolumn{2}{|c|}{ Intellectual level } \\
\hline Average & $34(27)$ \\
\hline Mild ID & 41 (33) \\
\hline Severe ID & $25(21)$ \\
\hline \multicolumn{2}{|c|}{ Expressive language } \\
\hline Verbal & $48(39)$ \\
\hline Nonverbal & $52(42)$ \\
\hline
\end{tabular}

ID, intellectual disability.

${ }^{\mathrm{a}}$ According to the ADOS calibrated severity score.

them accepted the evaluation phase, in which none of them were diagnosed with ASD. Finally, 30 out of 49 at risk children (60\%) were evaluated and seven met algorithm criteria on the ADOS-2 for an ASD diagnosis, confirmed by clinical evaluation. Two subjects did not meet cutoff for autism on the ADI-R. This result is not surprising, since the ADI-R, that is based on DSM-IV criteria (American Psychiatric Association, 2000), provides a dichotomic classification of autism or non-autism, without a cutoff for the broader category of ASD (Rutter et al., 2003b). Therefore, the ADI-R may not acknowledge more subtle forms of ASD (Saemundsen et al., 2003). Moreover, it is plausible that ADI-R scores are below the cutoff because the parents of these verbal, cognitively-able children were not worried about the neurodevelopment of their child: in fact, children have been referred for a clinical evaluation because of a positive ASD screening, and not because the parents were concerned. It is therefore possible that, in these high-functioning children, ASD symptoms may not be evident at 4.0-5.0 years of age (developmental period investigated in the ADI-R) and become manifest only when social demands exceed patients' limited capacities (DSM-5; American Psychiatric Association, 2013).

Figures 1 and 2 show the study flow for the screening and evaluation phases.

Probabilistic estimation was used to adjust the prevalence estimates for known non-response to the invitation for assessment within the two score bands where new cases were identified (see Baron-Cohen et al., 2009). Using the weightings of 10/6 (ten TN positive children who scored $\geqslant 15$ on the SCQ and six children in this score band who participated in the assessment) and of 17/ 10 (17 TN positive children who scored $11-14$ on the SCQ and ten children in this score band who participated in the assessment), the overall directly observed prevalence estimate for ASD was $[6 \times(10 / 6)]+[1 \times(17 / 10)]$, which corresponded to 11.7 new (undiagnosed) cases from the screened population.

Moreover, one child living in the geographical target area and attending a school which agreed to participate in the prevalence study arrived for clinical evaluation at the IRCCS Stella Maris Foundation independently from the ASDEU study. She was a 9-year-old girl (negative on the TN) whose parents were worried about her social difficulties, and she received a clinical diagnosis of ASD, which was also confirmed by the ASDEU team. We included this case in the prevalence study as arriving in 'another way'. Table 2 provides full assessment scores for the eight identified cases of ASD. All had an IQ in the normal range, between 93 and 118.

The prevalence for these new cases with no pre-existing ASD diagnosis ranged from $0.2 \%$ (95\% CI $0.06-0.33)$ only considering the evaluated cases, to $0.3 \%$ ( $95 \%$ CI $0.12-0.45)$ considering the probabilistic calculation.

\section{Summary of the prevalence estimate}

Considering the population of 10138 just using the number of children previously certified as affected by ASD plus the 15 whose diagnosis was modified to ASD by the ASDEU and the CNS teams, the prevalence (81 in 10138 ) was $0.8 \%$ (95\% CI $0.62-0.97$ ) that is $1 / 126$.

Considering the population of 4417 children (belonging to schools which accepted to participate in the TN/SCQ procedure) and using only the number of children certified with ASD (38 in 4417 ), the prevalence was $0.86 \%$ (95\% CI $0.59-1.13$ ) that is one in 116. As far as this population is concerned, the prevalence rises to $1 \%$ (95\% CI 0.74-1.34) if we consider the eight new cases identified in the population of children with no pre-existing diagnoses, and to $1.15 \%$ (95\% CI $0.83-1.46)$, that is one in 87 , if probabilistic calculation is considered.

\section{Discussion}

Thanks to the first European project (http://asdeu.eu/) designed to estimate ASD prevalence throughout Europe in children aged 7-9 years, our study reveals that one child out of 87 residing within the Province of Pisa (Italy) during 2016, has a DSM-5 diagnosis of ASD. To our knowledge, this is the first epidemiological study in Italy that evaluates the prevalence of ASD in children following a multi-stage methodology. The strengths of this study include the multi-step and multi-informant procedure adopted for ASD identification in a fairly large homogeneous area. Pisa is a province in Tuscany in Central Italy, with a mean annual income per inhabitant slightly higher than the Italian average (D'onofrio and Murro, 2013); in addition, there are no known risk or protective factors for ASD that might lead to variations in prevalence estimates in this province. Moreover, the province of Pisa has a tertiary care university hospital with a Unit specifically dedicated to the diagnosis and treatment of ASD children; therefore, we could assume that the propensity to seek health care for young subjects with ASD outside of the province of Pisa is minimal.

We used a multi-stage design for case identification, which consisted in: (a) consultation of existing records in education service provider databases for certified ASD diagnoses in children aged 7-9 years; (b) analysis of these records by means of a consensus between the ASDEU team and the child neuropsychiatry team responsible for the case, in order to verify the appropriateness of the neurodevelopmental diagnosis inserted into the database; (c) administration of two sequential screening tools (TN filled in by teachers and SCQ filled in by parents) with the aim of identifying the risk for ASD among 7-9-year-old undiagnosed children; (d) in-depth clinical evaluation with standard diagnostic instruments, such as the ADOS-2, of at-risk children by 


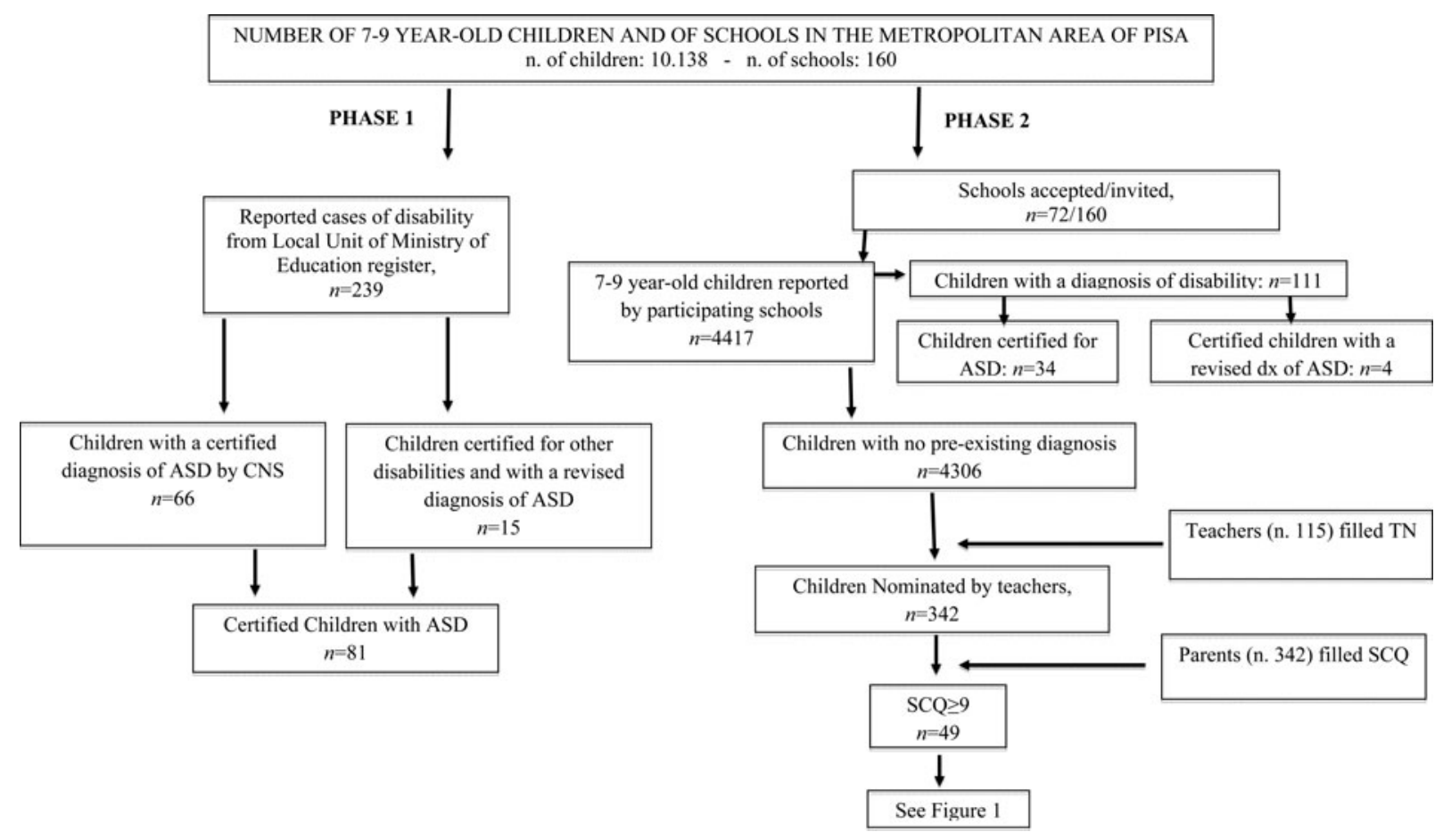

Fig. 1. Synopsis of Phase 1 (number of certified children with a diagnosis of ASD) and Phase 2 (number of potential new cases of ASD).

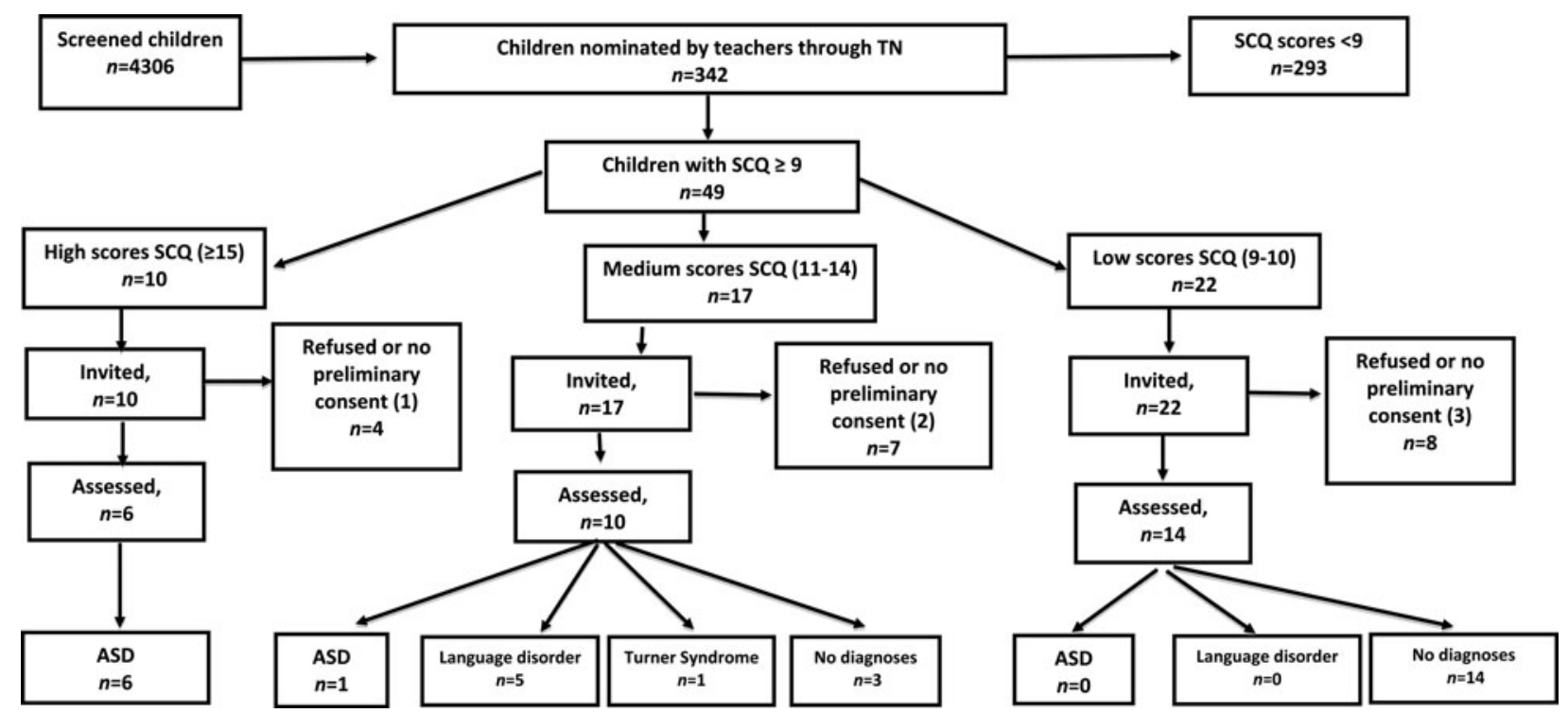

Fig. 2. Clinical assessment of children nominated by TN and with a SCQ score $\geqslant 9$.

professionals with over 15 years experience in assessing and diagnosing ASD. This methodology gave us a fairly complete picture of the distribution of autistic disorders within the spectrum.

The first two steps (that is phase 1 of the study) provided epidemiologic data regarding those ASD children who require help during their everyday life. In fact, all these children were certified as needing a support teacher, even if they showed good cognitive skills. The last two steps (that is phase 2 of the study: screening a typical school population) also enabled us to detect individuals with ASD who were not in contact with health services, and thus to increase the identification of ASD cases.

Using a similar procedure, Kim et al. (2011) screened the entire 7-12-year-old population of a South Korean community ( $n=36592$ children attending elementary schools plus $n=294$ children enrolled in the disability registry and special education schools) by administering the Autism Spectrum Screening Questionnaire (Ehlers et al., 1999) and a comprehensive ASD assessment battery (i.e. ADOS, ADI-R, cognitive tests) to screen- 
Table 2. Clinical characteristics of the new eight ascertained cases of ASD. For all cases, ADOS-2 Module 3 was used

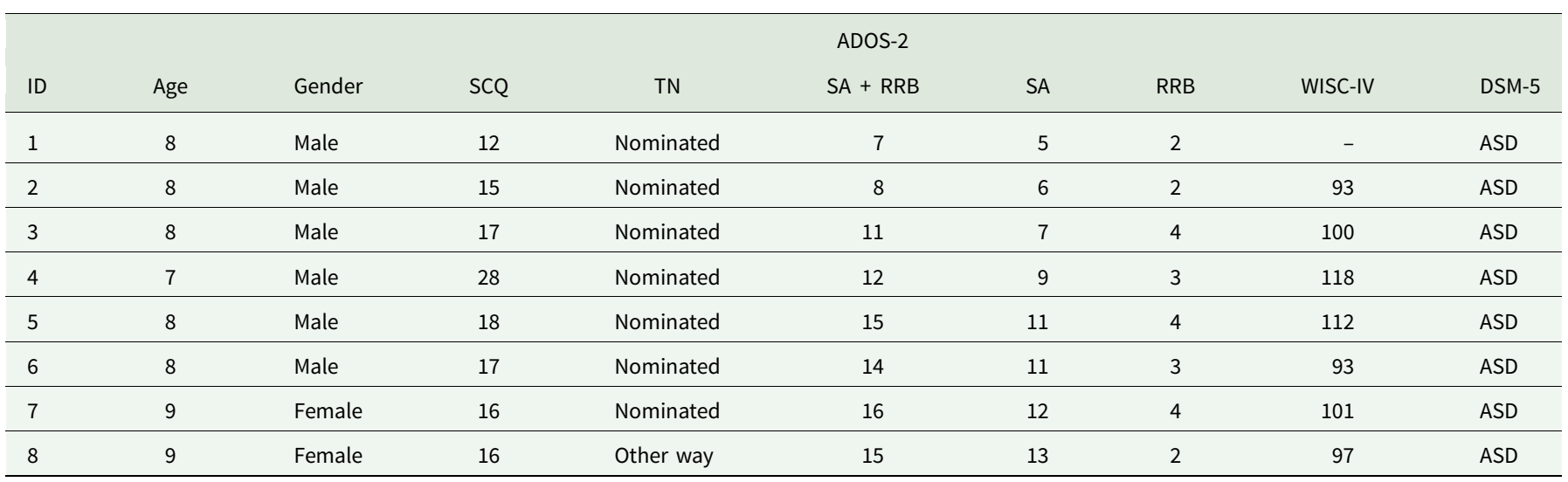

ADOS-2, Autism Diagnostic Observation Schedule-2; SCQ, Social Communication Questionnaire; TN, Teacher Nomination; SA, Social Affect; RRB, Restricted and Repetitive Behaviours; WISC-IV, Wechsler Intelligence Scale for Children, fourth edition; DSM-5, Diagnostic and Statistical Manual of Mental Disorders, fifth edition.

positive children. The authors found an ASD prevalence of $0.7 \%$ in the high-probability group (i.e. special education schools/disability registry), comparable with our group of certified children, and a large number of unidentified cases attending regular schools, with an ASD prevalence of $1.9 \%$ in this population.

Our results are consistent with those identified by Kim et al. (2011) as regards the prevalence estimate of the certified group $(0.8 \%$ in our study $v .0 .7 \%$ children enrolled in special education schools/disability registry in Kim's study). Also some characteristics of our sample were similar to those reported by Kim et al. (2011): for example, our male-to-female ratio is 5.2:1 $v$. the 5.1:1 found by Kim et al. (2011) and the percentage of children with severe intellectual disability was only slightly superior in our group than in Kim's study (25 v. 19\%). This prevalence of certified children is of particular importance, considering that Italy is one of the first European countries promoting school inclusion for ASD.

In contrast, significant differences emerge as far as ASD prevalence in the general population is concerned, in that the prevalence in our study $(0.3 \%)$ is fairly low compared with the one in Kim's study (1.9\%). A number of reasons could underlie these differences. First, we used a very conservative approach to diagnosis. In fact, only children with an ADOS-2 score above the cutoff for ASD and a DSM-5 diagnosis of ASD were considered as having ASD. Moreover, we used the DSM- 5 criteria, which are more restrictive than those of the DSM-IV used by Kim et al. (2011), who included also children with the controversial diagnosis of Pervasive Developmental Disorders Not Otherwise Specified. Indeed, $74 \%$ of cases in the general population sample were in the 'other ASD' category and not in the 'Autistic Disorder' category (Kim et al., 2011). The same problem occurs for the prevalence study by Baron-Cohen et al. (2009), which includes in the autism spectrum condition children with an ADOS score under the cutoff for the spectrum. Conversely, we only considered children with autism spectrum disorders, not conditions, and with an ADOS-2 score above the cutoff for ASD.

Nevertheless, we have to consider that we may have missed cases among screen-negative children on the TN or SCQ. This limit is reduced as far as TN is concerned by the demonstrated agreement (Hepburn et al., 2008) between the TN and the ASSQ, which has been used in many other studies, and as far as SCQ is concerned, by the fact that we considered the lower cutoff point of 9 instead of the official cutoff of 15. In particular, we cannot exclude an underestimation of females with autism. In fact, while in the study by Kim et al. (2011) the male-to-female ratio was $2.5: 1$, in our general population study it is $3.3: 1$. The fact that using our screening tools (TN and SCQ) only one female child received an ASD diagnosis, after clinical assessment, is in line with the gender ratio (8:1) of high-functioning ASD individuals (Hill et al., 2015), but it can also be an index of underidentification of females with ASD during screening procedures. Indeed, a further female child received an ASD diagnosis, but she screened negative on the TN. This poses the question of false negative female children who are on the spectrum. In regard to this issue, the literature indicates a gender bias in ASD diagnosis: in fact, girls with a normal intellectual level, who are not impaired by behavioural problems are less likely to receive an ASD diagnosis than boys, even at comparable levels of autistic symptoms (Dworzynski et al., 2012). In fact, females with ASD may have more socially accepted interests (Van WijngaardenCremers et al., 2014), and may be more likely to have developed coping strategies to manage social situations (Dean et al., 2016). Moreover, a larger proportion of verbally fluent females with ASD, compared with their male counterparts, receive scores in the non-ASD range on the ADOS-2 Module 3 algorithm, indicating that some females with ASD may be missed also by this instrument (Bishop et al., 2017).

Our findings have to be interpreted in the context of other limitations. First, we used first-level screening instruments (TN and SCQ) to determine which children needed further evaluation to determine an ASD diagnosis. The administration of two sequential screening tools (TN and SCQ) aimed to increase the specificity (i.e. to reduce false positives): in fact, this sequential procedure involved applying a second screening test (SCQ) to children who initially screened positive (TN). In this way, an erroneous positive identification with consequent family stress as well as time-consuming assessment procedures is avoided (Charman and Gotham, 2013). Nevertheless, an underestimation of ASD, particularly in high-functioning subjects who do not give teachers and/or parents cause for concern cannot be excluded. In fact, teachers and parents may be focused mainly on academic achievements, and fail to recognise or minimise the socio-communicative difficulties as well as the peculiar and restrictive interests of these children. In accordance with this view, recent literature suggests there may be a delay in obtaining an ASD diagnosis for children with average IQ scores (Jónsdóttir et al., 2011; Mazurek et al., 
2014; Romhus et al., 2017). These individuals might receive a first ASD diagnosis in adolescence or adulthood, or may be referred to mental health services, not for core ASD symptoms, but for comorbid psychiatric disorders (Aggarwal and Angus, 2015), which are frequent in high-functioning adolescents (Strang et al., 2012) and adults (Lugnegård et al., 2011) with ASD. Other high-functioning subjects could be incorrectly diagnosed (Lugnegård et al., 2012; Takara et al., 2015) or never be diagnosed with an ASD (Brugha et al., 2011) and this evidence leads respectively to inappropriate treatment or missed opportunities to treat the disorder.

Second, another possible source of ASD prevalence underestimation is the absence of parental consent for further evaluation of all the children screened positive on the TN and SCQ. Therefore, some children, despite being at risk of ASD, did not receive a clinical assessment for suspected ASD. One possible explanation for parental refusal of a direct clinical evaluation requiring both parent and child attendance is the presence of logistical difficulties (e.g. taking time off work, transportation, childcare for sibling/s). Another possibility is that, despite increasing public awareness and decreasing stigmatisation surrounding ASD in recent years (Kapp et al., 2013; DeVilbiss and Lee, 2014), some parents could be reluctant to accept an in-depth neuropsychiatric evaluation for their intelligent child, and therefore they prefer to refuse the diagnostic assessment.

Third, an additional cause of underdiagnosis could derive from the absence of further evaluation among children who screen negative on the TN or SCQ. Indeed, the TN, despite being a time-efficient and cost-effective way to screen children in schools (Hepburn et al., 2008), is certainly less able to detect ASD than more universal screening procedures, which can identify subjects who have symptoms that are subtle, subthreshold or attributed to other conditions (Schanding et al., 2012). Analogously, the SCQ is an acceptable screening instrument for $\mathrm{ASD}$, and its accuracy is enhanced when the Lifetime version is used in a population older than 4 years of age (Chesnut et al., 2017), as in our sample. However, even if we chose a relatively inclusive SCQ cut-off score (i.e., able to identify the majority of individuals with ASD, but also including subjects who are not), we may still have missed cases among children with an SCQ score <9. Indeed, Barnard-Brak et al. (2016) calculated that using a cutoff of 7 on the SCQ, 5.8\% of children were incorrectly identified as not having ASD (e.g., false negatives). For this reason, some authors have suggested lowering the cutoff score to 8 or 7 in order to detect high-functioning subjects (Schanding et al., 2012), or to a score of 7 for school-aged elementary individuals (Barnard-Brak et al., 2016).

Overall, the aspects above-mentioned may have contributed to the lower ASD prevalence obtained in comparison with other studies conducted in other countries. However, one of the strengths of our study is that it provides the most up-to-date epidemiological investigation of ASD in Italy and that it is one of the first investigations using the strict DSM-5 criteria for ASD (Morales-Hidalgo et al., 2018). Another strength is related to the procedure that is different from other prevalence studies in which ASD cases have been identified based on existing health records (Taylor et al., 2013), service provider databases (Croen et al., 2002), or on parent report alone (Blumberg, et al., 2013; Zablotsky et al., 2015), without any additional direct diagnostic clinical evaluation. Even compared with the studies that used a similar diagnostic methodology to ours (e.g., Baron-Cohen et al., 2009), the final criteria we used for assigning an ASD diagnosis to children found positive on the TN and SCQ were more stringent as all subjects had to meet ASD cutoffs on the ADOS-2 Module 3 algorithm, in addition to the DSM-5 diagnostic criteria for ASD. In the study by Baron-Cohen et al. (2009) instead, not only disorders but also conditions were considered.

In conclusion, this study indicates a prevalence of ASD in 7- to 9 -year-old children living in a defined large catchment area in Italy, of about one in 87 . This prevalence covers a wide spectrum of autistic disorders using DSM-5 criteria, from children with a clear diagnosis (who in Italy attend regular schools with a support teacher) to children with no pre-existing ASD diagnoses. This result indicates the need to implement, in line with the estimated prevalence, specialised services and multidisciplinary ASD teams (within the public healthcare system) able to address the varied needs (in term of diagnosis, rehabilitative intervention and care) of the different autisms within the spectrum.

Acknowledgements. The Autism Spectrum Disorders in the European Union project - ASDEU has been funded by the DG-SANTÉ, European Commission [grant number SANCO/2014/C2/035]. This work was also supported by a grant from the IRCCS Fondazione Stella Maris (Ricerca Corrente, and the ' $5 \times 1000$ ' voluntary contributions, Italian Ministry of Health).

Conflict of interest. No author has any conflicts of interest to declare.

Ethical standards. The authors assert that all procedures contributing to this work comply with the ethical standards of the relevant national and institutional committees on human experimentation and with the Helsinki Declaration of 1975, as revised in 2008.

Availability of data and materials. The data collected in the present study contain sensitive information on a very vulnerable group, i.e., children with ASD. Even though the data have been anonymised, they still contain enough details to enable identification of single individuals. Therefore, in order to approve the study, the Regional Ethical Review Board in Florence (Tuscany) made considerable restrictions regarding data access. This means we will not be able to share our data with other researchers outside the ASDEU group.

\section{References}

Aggarwal S and Angus B (2015) Misdiagnosis versus missed diagnosis: diagnosing autism spectrum disorder in adolescents. Australasian Psychiatry 23, $120-123$.

Allen CW, Silove N, Williams K and Hutchins P (2007) Validity of the Social Communication Questionnaire in assessing risk of autism in preschool children with developmental problems. Journal of Autism and Developmental Disorders 37, 1272-1278.

American Psychiatric Association (2000) Diagnostic and statistical manual of mental disorders (4th ed, text revision). American Psychiatric Association, Washington, DC.

American Psychiatric Association (2013) Diagnostic and Statistical Manual of Mental Disorders (DSM-5), 5th Edn. Washington, DC: American Psychiatric Publishing.

Arvidsson O, Gillberg C, Lichtenstein P and Lundström S (2018) Secular changes in the symptom level of clinically diagnosed autism. Journal of Child Psychology and Psychiatry 59, 744-751.

Bachmann C, Gerste B and Hoffmann F (2018) Diagnoses of autism spectrum disorders in Germany: time trends in administrative prevalence and diagnostic stability. Autism 22, 283-290.

Baio J, Wiggins L, Christensen DL, Maenner MJ, Daniels J, Warren Z, Kurzius-Spencer M, Zahorodny W, Robinson Rosenberg C, White T, Durkin MS, Imm P, Nikolaou L, Yeargin-Allsopp M, Lee LC, Harrington R, Lopez M, Fitzgerald RT, Hewitt A, Pettygrove S, Constantino JN, Vehorn A, Shenouda J, Hall-Lande J, Van Naarden Braun K and Dowling NF (2018) Prevalence of Autism Spectrum Disorder among children aged 8 years - Autism and developmental 
disabilities monitoring network, 11 sites, United States, 2014. Morbidity and Mortality Weekly Report. Surveillance Summaries 67, 1-23.

Barnard-Brak L, Brewer A, Chesnut S, Richman D and Schaeffer AM (2016) The sensitivity and specificity of the social communication questionnaire for autism spectrum with respect to age. Autism Research 9, 838-845.

Baron-Cohen S, Scott FJ, Allison C, Williams J, Bolton P, Matthews FE and Brayne C (2009) Prevalence of autism-spectrum conditions: UK schoolbased population study. British Journal of Psychiatry 194, 500-509.

Bishop SL, Sweeney MP, Huerta M, Havdahl A and Lord C (2017) Sex differences in ADOS-2 scores and classifications among verbally fluent children with Autism Spectrum Disorder. Poster presented at the annual International Meeting for Autism Research.

Blumberg SJ, Bramlett MD, Kogan MD, Schieve LA and Lu MC (2013) Changes in prevalence of parent-reported autism spectrum disorder in school-aged US children: 2007 to 2011-2012. National Health Statistics Reports 65, 1-11.

Boilson AM, Staines A, Ramirez A, Posada M and Sweeney MR (2016) Operationalisation of the European Protocol for Autism Prevalence (EPAP) for Autism Spectrum disorder prevalence measurement in Ireland. Journal of Autism and Developmental Disorders 46, 3054-3067.

Brugha TS, McManus S, Bankart J, Scott F, Purdon S, Smith J, Bebbington P, Jenkins R and Meltzer H. (2011) Epidemiology of autism spectrum disorders in adults in the community in England. Archives of General Psychiatry 68, 459-465.

Centers for Disease Control and Prevention (2007a) Prevalence of autism spectrum disorders- autism and developmental disabilities monitoring network, six sites, United States, 2000. Morbidity and Mortality Weekly Report. Surveillance Summaries 56, 1-11.

Centers for Disease Control and Prevention (2007b) Prevalence of autism spectrum disorders - autism and developmental disabilities monitoring network, 14 sites, United States, 2002. Morbidity and Mortality Weekly Report. Surveillance Summaries 56, 12-28.

Centers for Disease Control and Prevention (2009) Prevalence of autism spectrum disorders - autism and developmental disabilities monitoring network, United States, 2006. Morbidity and Mortality Weekly Report. Surveillance Summaries 58, 1-20.

Centers for Disease Control and Prevention (2012) Prevalence of autism spectrum disorders- autism and developmental disabilities monitoring network, 14 sites, United States, 2008. Morbidity and Mortality Weekly Report. Surveillance Summaries 61, 1-19.

Centers for Disease Control and Prevention (2014) Prevalence of autism spectrum disorders among children aged 8 years- autism and developmental disabilities monitoring network, 11 sites, United States, 2010. Morbidity and Mortality Weekly Report. Surveillance Summaries 63, 1-22.

Charman T and Gotham K (2013) Measurement issues: screening and diagnostic instruments for ASD. Lessons from research and practice. Child and Adolescent Mental Health 18, 52-63.

Chesnut SR, Wei T, Barnard-Brak L and Richman DM (2017) A metaanalysis of the social communication questionnaire: screening for autism spectrum disorder. Autism 21, 920-928.

Christensen DL, Baio J, Van Naarden Braun K, Bilder D, Charles J, Constantino JN, Daniels J, Durkin MS, Fitzgerald RT, KurziusSpencer M, Lee LC, Pettygrove S, Robinson C, Schulz E, Wells C, Wingate MS, Zahorodny W and Yeargin-Allsopp M (2016) Centers for disease control and prevention (CDC). prevalence and characteristics of autism Spectrum disorder Among children aged 8 years--autism and developmental disabilities monitoring network, 11 sites, United States, 2012. Morbidity and Mortality Weekly Report. Surveillance Summaries 65, 1-23.

Corsello C, Hus V, Pickles A, Risi S, Cook EH, Leventhal BL and Lord C (2007) Between a ROC and a hard place: decision making and making decisions about using the SCQ. Journal of Child Psychology and Psychiatry 48, 932-940.

Croen L, Grether JK, Hoogstrate J and Selvin S (2002) The changing prevalence of autism in California. Journal of Autism and Developmental Disorders 32, 207-215.

Davidovitch M, Hemo B, Manning-Courtney P and Fombonne É (2013) Prevalence and incidence of autism spectrum disorder in an Israeli population. Journal of Autism and Developmental Disorders 43, 785-793.
Dereu M, Warreyn P, Raymaekers R, Meirsschaut M, Pattyn G, Schietecatte I and Roeyers H (2010) Screening for autism spectrum disorders in Flemish day-care centres with the checklist for early signs of developmental disorders. Journal of Autism and Developmental Disorders 40, 1247-1258.

Dean M, Harwood R and Kasari C (2017) The art of camouflage: Gender differences in the social behaviors of girls and boys with autism spectrum disorder. Autism 21, 678-89.

DeVilbiss EA and Lee BK (2014) Brief report: trends in US National autism awareness from 2004 to 2014: the impact of national autism awareness month. Journal of Autism and Developmental Disorders 44, 3271-3273.

D'onofrio A and Murro P (2013) Local banking development and income distribution across Italian provinces. CASMEF Working Paper 7, 1-34. doi: $10.2139 /$ ssrn.2350165.

Dworzynski K, Ronald A, Bolton P and Happé F (2012) How different are girls and boys above and below the diagnostic threshold for ASD? Journal of American Academy Child and Adolescent Psychiatry 51, 788-797.

Ehlers S, Gillberg C and Wing L (1999) A screening questionnaire for Asperger syndrome and other high-functioning autism spectrum disorders in school age children. Journal of Autism and Developmental Disorders 29, 129-141.

Elsabbagh M, Divan G, Koh YJ, Kim YS, Kauchali S, Marcín C, MontielNava C, Patel V, Paula CS, Wang C, Yasamy MT and Fombonne E (2012) Global prevalence of autism and other pervasive developmental disorders. Autism Research 5, 160-179.

Ferrante M, Barone R, Fazio A, Zerbo S, Margherita V, Rizzo R, Fichera G and Fiore M (2015) Prevalence and age at diagnosis of Autism Spectrum Disorder in south Italy, 2004-2014. The European Journal of Public Health 25(suppl. 3). DOI: 10.1093/eurpub/ckv175.099

Fombonne E (2009) Epidemiology of pervasive developmental disorders. Pediatric Research 65, 591-598.

Gotham K, Risi S, Pickles A and Lord C (2007) The Autism Diagnostic Observation Schedule: revised algorithms for improved diagnostic validity. Journal of Autism and Developmental Disorders 37, 613-627.

Gotham K, Pickles A and Lord C (2009) Standardizing ADOS scores for a measure of severity in autism spectrum disorders. Journal of Autism and Developmental Disorders 39, 693-705.

Hansen SN, Schendel DE and Parner ET (2015) Explaining the increase in the prevalence of autism spectrum disorders: the proportion attributable to changes in reporting practices. JAMA Pediatrics 169, 56-62.

Hepburn SL, DiGuiseppi C, Rosenberg S, Kaparich K, Robinson C and Miller L (2008) Use of a teacher nomination strategy to screen for autism spectrum disorders in general education classrooms: a pilot study. Journal of Autism and Developmental Disorders 38, 373-382.

Hertz-Picciotto I, Schmidt RJ and Krakowiak P (2018) Understanding environmental contributions to autism: causal concepts and the state of science. Autism Research 11, 554-586.

Hill AP, Zuckerman K and Fombonne É (2015) Epidemiology of Autism Spectrum Disorders. In (Maria de los Angeles Robinson-Agramonte, ed.) Translational Approaches to Autism Spectrum Disorder. Springer, pp. 13-38.

Idring S, Lundberg M, Sturm H, Dalman C, Gumpert C, Rai D, Lee BK and Magnusson C (2015) Changes in prevalence of ASD in 2001-2011: findings from the Stockholm youth cohort. Journal of Autism and Developmental Disorders 45, 1766-1773.

Jónsdóttir SL, Saemundsen E, Antonsdóttir IS, Sigurdardóttir S and Ólason D (2011) Children diagnosed with ASD before or after the age of 6 years. Research in Autism Spectrum Disorders 5, 175-184.

Kapp SK, Gillespie-Lynch K, Sherman LE and Hutman T (2013) Deficit, difference, or both? Autism and neurodiversity. Developmental Psychology 49, 59-71.

Kim YS, Leventhal BL, Koh Y-J, Fombonne É, Laska E, Lim EC, Cheon KA, Kim SJ, Kim YK, Lee H, Song DH and Grinker RR (2011) Prevalence of autism spectrum disorders in a total population sample. American Journal of Psychiatry 168, 904-912.

Kočovská E, Biskupstø R, Carina Gillberg I, Ellefsen A, Kampmann H, Stórá T, Billstedt E and Gillberg C (2012) The rising prevalence of autism: a prospective longitudinal study in the Faroe Islands. Journal of Autism and Developmental Disorders 42, 1959-1966.

Lord C, Rutter M, DiLavore PC, Risi S, Gotham K and Bishop S (2012) Autism Diagnostic Observation Schedule 2nd Edn. Torrance, CA: Western Psychological Services. 
Lugnegård T, Hallerbäck MU and Gillberg C (2011) Psychiatric comorbidity in young adults with a clinical diagnosis of Asperger syndrome. Research in Developmental Disabilities 32, 1910-1917.

Lugnegård T, Hallerbäck MU and Gillberg C (2012) Personality disorders and autism spectrum disorders: what are the connections? Comprehensive Psychiatry 53, 333-340.

Mazurek MO, Handen BL, Wodka EL, Nowinski L, Butter E and Engelhardt CR (2014) Age at first autism spectrum disorder diagnosis: the role of birth cohort, demographic factors, and clinical features. Journal of Developmental and Behavioral Pediatrics 35, 561-569.

Modabbernia A, Velthorst E and Reichenberg A (2017) Environmental risk factors for autism: an evidence-based review of systematic reviews and meta-analyses. Molecular Autism 8, 13.

Morales-Hidalgo P, Roigé-Castellví J, Hernández-Martínez C, Voltas N and Canals J (2018) Prevalence and characteristics of Autism spectrum disorder among Spanish school-age children. Journal of Autism and Developmental Disorders 48, 3176-3190.

Nevison CD and Blaxill M (2017) Diagnostic substitution for intellectual disability: a flawed explanation for the rise in Autism. Journal of Autism and Developmental Disorders 47, 2733-2742.

Parker-Athill EC and Tan J (2010) Maternal immune activation and autism spectrum disorder: interleukin-6 signaling as a key mechanistic pathway. Neurosignals 18, 113-128.

Parner ET, Thorsen P, Dixon G, de Klerk N, Leonard H, Nassar N, Bourke J, Bower C and Glasson EJ (2011) A comparison of autism prevalence trends in Denmark and Western Australia. Journal of Autism and Developmental Disorders 41, 1601-1608.

Parsons VL, Moriarity C, Jonas K, Moore TF, Davis KE and Tompkins L (2014) Design and estimation for the National Health Interview Survey, 2006-2015. Vital and Health Statistics. Series 2, Data Evaluation and Methods Research 165, 1-53.

Patel S, Masi A, Dale RC, Whitehouse AJO, Pokorski I, Alvares GA, Hickie IB, Breen E, Guastella AJ (2017) Social impairments in autism spectrum disorder are related to maternal immune history profile. Molecular Psychiatry doi: 10.1038/mp.2017.201. [Epub ahead of print]

Ramsey E, Kelly-Vance L, Allen JA, Rosol O and Yoerger M (2016) Autism spectrum disorder prevalence rates in the United States: methodologies, challenges, and implications for individual states. Journal of Developmental and Physical Disabilities 28, 803-820.

Rice CE, Rosanoff M, Dawson G, Durkin MS, Croen LA, Singer A and Yeargin-Allsopp M. (2013) Evaluating changes in the prevalence of ASD. Public Health Review 34, 1-22.

Romhus S, Herder GA, Grindheim E, Schjølberg S and Howlin P (2017). Changes in the frequency and characteristics of children diagnosed with autistic disorder in two Norwegian cohorts: 1992 and 2009. Scandinavian Journal of Child and Adolescent Psychiatry and Psychology 5, 3-12.

Rutter M, Bailey A and Lord L (2003a) The Social Communication Questionnaire. Los Angeles: Western Psychological Services.

Rutter M, Le Couteur A and Lord C (2003b) ADI-R. Autism Diagnostic Interview Revised. Manual. Los Angeles: Western Psychological Services.
Saemundsen E, Magnússon P, Smári J and Sigurdardóttir S (2003) Autism diagnostic interview-revised and the childhood Autism rating scale: convergence and discrepancy in diagnosing autism. Journal of Autism and Developmental Disorders 33, 319-328.

Saemundsen E, Magnússon P, Georgsdóttir I, Egilsson E and Rafnsson V (2013) Prevalence of autism spectrum disorders in an Icelandic birth cohort. BMJ Open 3, e002748.

Schanding GTJ, Nowell KP and Goin-Kochel RP (2012) Utility of the social communication questionnaire-current and social responsiveness scale as teacher-report screening tools for autism spectrum disorders. Journal of Autism and Developmental Disorders 42, 1705-1716.

Shelton JF, Tancredi DJ and Hertz-Picciotto I (2010) Independent and dependent contributions of advanced maternal and paternal ages to autism risk. Autism Research 3, 30-39.

Skonieczna-Żydecka K, Gorzkowska I, Pierzak-Sominka J and Adler G (2016) The prevalence of ASD in West Pomeranian and Pomeranian regions of Poland. Journal of Applied Research in Intellectual Disabilities. doi: 10.1111/jar.12238. [Epub ahead of print].

Snow AV and Lecavalier L (2008) Sensitivity and specificity of the modified checklist for autism in toddlers and the social communication questionnaire in preschoolers suspected of having pervasive developmental disorders. Autism 12, 627-644.

Strang JF, Kenworthy L, Daniolos P, Case L, Wills MC, Martin A and Wallace GL (2012) Depression and anxiety symptoms in children and adolescents with ASD without intellectual disability. Research in Autism Spectrum Disorders 6, 406-412.

Takara K, Kondo T and Kuba T (2015) How and Why is ASD misdiagnosed in adult patients? From diagnostic problem to management for adjustment. Mental Health in Family Medicine 11, 73-88.

Taylor B, Jick H and MacLaughlin D (2013) Prevalence and incidence rates of autism in the UK: time trend from 2004-2010 in children aged 8 years. BMJ OPEN 3, e003219.

Van Bakel MM, Delobel-Ayoub M and Cans C (2015) Low but increasing prevalence of ASD in a French area from register-based data. Journal of Autism and Developmental Disorders 45, 3255-3261.

Van Wijngaarden-Cremers PJ, van Eeten E, Groen WB, Van Deurzen PA, Oosterling IJ and Van der Gaag RJ (2014) Gender and age differences in the core triad of impairments in autism spectrum disorders: a systematic review and meta-analysis. Journal of Autism and Developmental Disorders 44, 627-635.

Wechsler D (2003) Wechsler Intelligence Scale for Children - Fourth Edition (WISC-IV). San Antonio, TX: Pearson Education Inc.

Wu S, Wu F, Ding Y, Hou J, Bi J and Zhang Z (2017) Advanced parental age and autism risk in children: a systematic review and meta-analysis. Acto Psychiatrica Scandinavica 135, 29-41.

Xu G, Strathearn L, Liu B and Bao W (2018) Prevalence of ASD Among US children and adolescents 2014-2016. JAMA 319, 81-82.

Zablotsky B, Black L, Maenner MJ, Schieve LA and Blumberg SJ (2015) Estimated prevalence of Autism and other developmental disabilities following questionnaire changes in the 2014 National Health Interview Survey. National Health Statistics Reports 87, 1-2. 